\title{
Large subgaleal hematoma caused by valproic acid: a case report
}

\author{
Aldo José F da Silva ${ }^{1}$, Ana Carolina Ruela Carvalho ${ }^{2}$, Fabiana Lopes Amaral ${ }^{2}$, Auxiliadora \\ Damianne Pereira Vieira da Costa ${ }^{2}$
}

Received: 21July 2020 / Published: 06 December 2020

\begin{abstract}
Introduction: Valproic acid (VPA), used to treat epilepsy, may present hematological abnormalities as complications. One such example is bleeding associated with trauma. We herein report a case about large subgaleal hemorrhage that occurred after a mild head trauma in a child following VPA administration. Case Report: A 12-year-old female child diagnosed with Rett Syndrome had difficulty in controlling epilepsy and was taking $50 \mathrm{mg} / \mathrm{kg} /$ day VPA. After suffering from a mild head trauma, a large subgaleal hematoma was evidenced on Computed Tomography(CT) scan of head. Surgical intervention was performed, with drainage of the hematoma, and the patient showed progression. Discussion: The incidence of coagulopathy related to VPA is $4 \%$, and thrombocytopenia is the most common coagulation disorder. Hemorrhagic complications can occur regardless of the plasma levels or duration of treatment. In VPA therapy, the side effects should be considered, particularly hematological abnormalities. In our case, an unusual hemorrhagic complication (subgaleal hematoma) was reported, with good surgical resolution of the pathology.
\end{abstract}

Key words: Valproic acid, Rett Syndrome, hematoma, epilepsy, thrombocytopenia

1 Division of Pediatric Neurosurgery, General State Hospital (GSH), Maceió, AL, Brazil

2 Pediatric Division, General State Hospital (GSH), Maceió, AL,Brazil

To whom correspondence should be addressed: Aldo José F. da Silva, MD, [E-mail: neuroajfs@yahoo.com.br]

Journal homepage: www.sbnped.com.br

\section{Introduction}

Valproic acid (VPA) is an anticonvulsant used to treat a broad spectrum of focal and generalized epilepsies; it also demonstrates benefits in psychiatric pathology. The effects of VPA are varied including teratogenicity, amenorrhea, weight gain, hair loss, nausea, tremors, liver toxicity, and pancreatitis [1-3]. Hematological abnormalities are also common such as thrombocytopenia, abnormal platelet function, reduced plasma fibrinogen concentration, vitamin Kdependent factors, Von Willebrand factor, and factor XIII. There are reports of intracranial hemorrhage, pulmonary bleeding, gastrointestinal bleeding, bleeding associated with surgeries, and trauma in patients using VPA [4-6].

The report presented here referred to a child with epilepsy using VPA, who developed a large subgaleal hematoma after suffering a mild head trauma.

\section{Case Report}

A 12-year-old female child diagnosed with Rett syndrome presented with refractory seizures. She was under treatment with valproic acid (VPA) for more than 5 years, and at the time of the events in this report, the dose taken was 2,250 $\mathrm{mg}(50 \mathrm{mg} / \mathrm{kg} /$ day). Thirty days before admission, she suffered a fall without loss of consciousness; however, presenting a progressive bulging of the scalp (Figure 2a). A skull tomography showed a massive fronto-temporo-parieto-occipital subgaleal hematoma (Figure 1a-c) with no signs of cranial fracture (Figure 1d). She was diagnosed with anemia and thrombocytopenia, with a platelet count of $69 \times 103 / \mathrm{mm} 3$, International Normalized Ratio (INR) of 1.15, and 


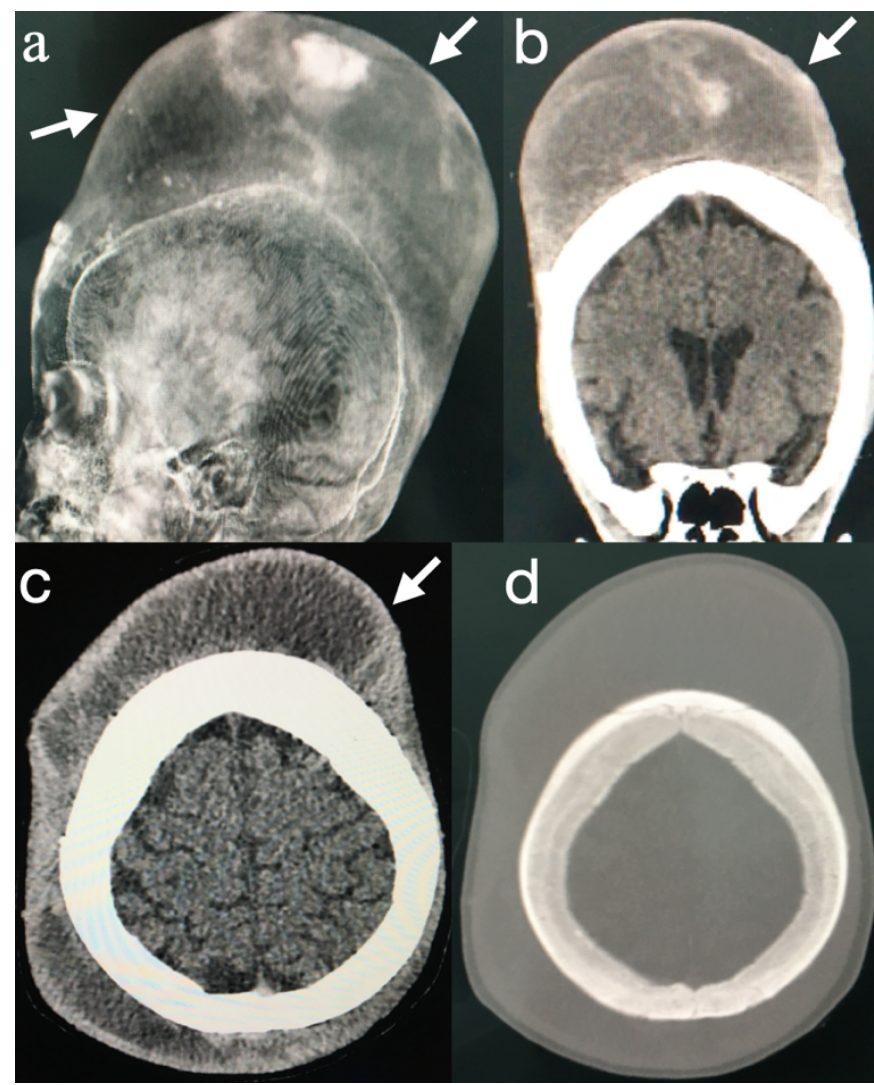

Figure 1- 3D CT scan (a), coronal CT scan (b) and axial CT scan (c) showing large subgaleal hematoma (white arrows); bone window (d) without skull fracture

activated partial thromboplastin time of $21.10 \mathrm{~s}$. Transfusion of red blood cell concentrate and fresh plasma was performed. Seven days after replacing VPA to another anticonvulsant (phenobarbital), the platelet count improved (264 $\times 103 / \mathrm{mm} 3$ ), and the child was considered able to undergo a surgical procedure. Hematological anomalies were investigated, but none were found. First, an aspiration puncture was tried; however, it was unsuccessful. It was subsequently decided to perform open drainage through a biparietal zigzag incision, with drainage of $600 \mathrm{~mL}$ of subgaleal hematoma (Figure $2 \mathrm{~b}$ and c). A suction drain (Figure 2d) was left in the subgaleal space and removed 48 hours after the surgical procedure. In the postoperative period, the patient evolved well, and on the sixth day she was discharged from the hospital. She remained in use of phenobarbital only, at $200 \mathrm{mg} / \mathrm{day}$, without further seizures.

\section{Discussion}

Rett syndrome is a childhood neurodevelopmental disorder first described in 1966 by Andreas Rett. It affects girls more often and is characterized by

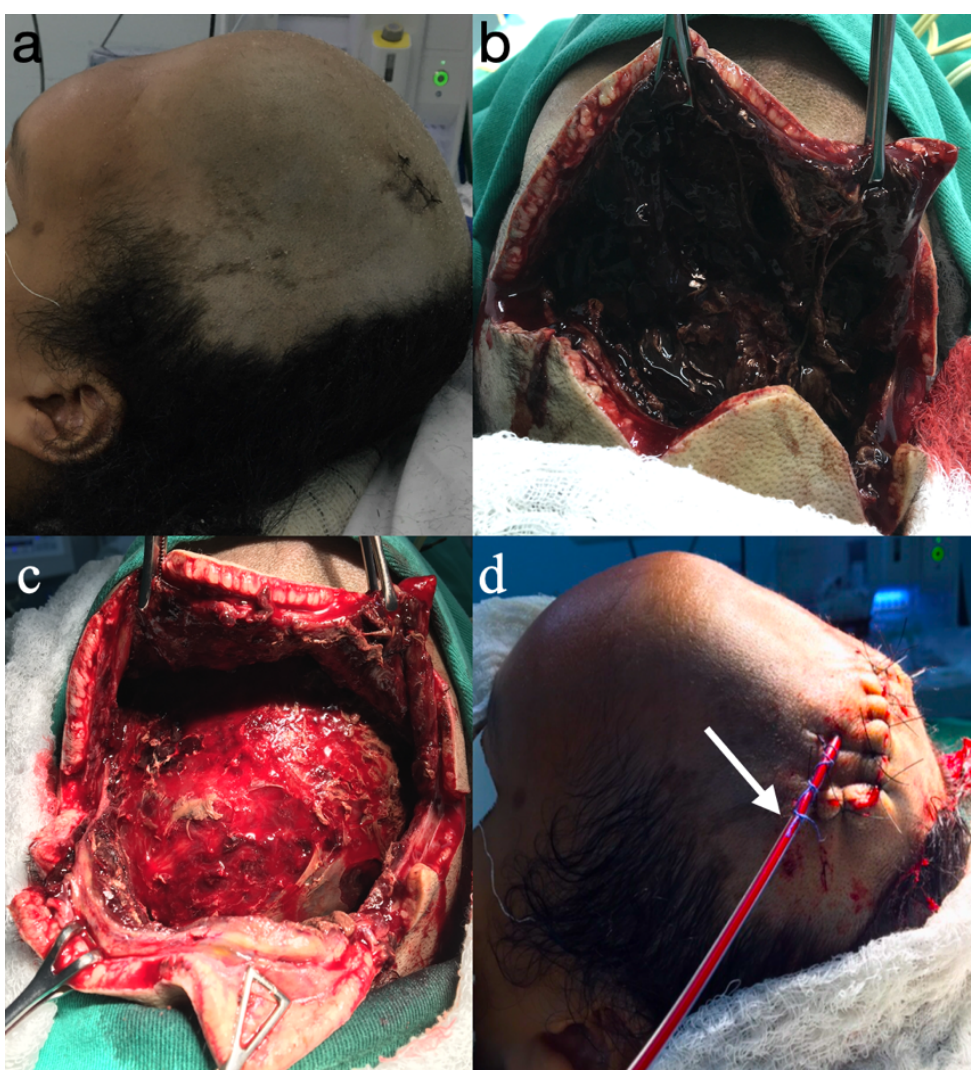

Figure 2 - Bulge on scalp (a); Subgaleal hematoma (b); Hematoma evacuation (c); Postoperative with suction drain (d)

regression of acquired motor skills, loss of acquired spoken language, hand stereotypies, and seizures. Epilepsy is present in most patients $(60 \%-80 \%)$, and it is refractory to anticonvulsants in a third of the cases. One of the most commonly used drugs is valproic acid (VPA) [7].

The synthesis of VPA was described in 1882 by Burton, an American chemist. VPA is derived from valeric acid, a component of the plant Valeriana officinalis [8, 9]. VPA is mostly metabolized by the liver, and its mechanism of action is the inhibition of $\mathrm{N}$ methyl D-aspartate mediated excitation, potentiating the inhibitory activity of gamma-aminobutyric acid, and blocking the voltage-dependent sodium, calcium, and potassium channels $[9,10]$.

The incidence of VPA-related coagulopathy is $4 \%$ [1]. Thrombocytopenia is one of the most common coagulation disorders, with an incidence of $5 \%-60 \%$. Children are more affected than adults, and a possible explanation is the need for higher doses of VPA to often control refractory seizures [5]. Patients taking VPA suffer increased blood loss during surgery compared to those not taking VPA, although there is no significant difference in bleeding time and PT/aPTT [11]. Other studies, however, have not confirmed an association between VPA use and increased bleeding during surgical intervention [12]. In fact, there is a great controversy regarding the hematological 
abnormalities caused by VPA and the mechanism of the resulting coagulopathy [13].

There are some hypotheses to explain thrombocytopenia: 1) immunological processes with VPA inducing the formation of platelet autoantibodies and 2) bone marrow toxicity, with pancytopenia, bone marrow suppression, abnormal megakaryocytes, erythrocyte precursors, macrocytosis, and neutrophil anomalies [5, 14]. Laboratory changes and hemorrhagic complications can occur independent of VPA plasma level and duration of treatment [15].

In our reported case, the child had a long-term use of VPA, causing thrombocytopenia, without any other laboratory alterations. The child suffered a mild brain trauma that was sufficient to cause large subgaleal bleeding.

VPA therapy is suggested for the treatment of various types of epilepsy. However, its side effects must be considered, including hematological abnormalities. In our case, the patient presented an unusual type of hemorrhagic complication (subgaleal hematoma) with good resolution of the problem.

\section{Disclosure Statement}

The authors have no conflicts of interest to declare.

\section{Funding statement}

None.

\section{References}

1. Gerstner T, Teich M, Bell N, et al. Valproateassociated coagulopathies are frequent and variable in children. Epilepsia. 2006;47(7):11361143. doi:10.1111/j.1528-1167.2006.00587.x

2. Vasudev K, Keown P, Gibb I, McAllister-Williams $\mathrm{RH}$. Hematological effects of valproate in psychiatric patients: what are the risk factors?. J Clin Psychopharmacol. 2010;30(3):282-285. doi:10.1097/JCP.0b013e3181db2684

3. Cannizzaro E, Albisetti M, Wohlrab G, Schmugge $M$. Severe bleeding complications during antiepileptic treatment with valproic acid in children. Neuropediatrics. 2007;38(1):42-45. doi:10.1055/s-2007-981448

4. Besag FM. Abnormal bleeding or bruising associated with antieplieptic treatment. Curr Drug Saf. 2009;4(3):167-168. doi:10.2174/157488609789006976

5. Koenig S, Gerstner T, Keller A, Teich M, Longin $E$, Dempfle CE. High incidence of vaproateinduced coagulation disorders in children receiving valproic acid: a prospective study. Blood Coagul Fibrinolysis. 2008;19(5):375-382. doi:10.1097/MBC.0b013e3282f3f9ec

6. Bhaskar S, Sobti S, Singh AK. Massive scalp hematoma: An unusual presentation of valproic acid toxicity. Ann Indian Acad Neurol.
2012;15(4):315-316. doi:10.4103/09722327.104346

7. Krajnc N. Management of epilepsy in patients with Rett syndrome: perspectives and considerations. Ther Clin Risk Manag. 2015;11:925-932. doi:10.2147/TCRM.S55896

8. Buoli M, Serati M, Botturi A, Altamura AC. The Risk of Thrombocytopenia During Valproic Acid Therapy: A Critical Summary of Available Clinical Data. Drugs R D. 2018;18(1):1-5. doi:10.1007/s40268-017-0224-6

9. Kumar R, Vidaurre J, Gedela S. Valproic AcidInduced Coagulopathy. Pediatr Neurol. 2019;98:25-30. doi:10.1016/j.pediatrneurol.2019.04.019

10. Chateauvieux S, Morceau F, Dicato M, Diederich M. Molecular and therapeutic potential and toxicity of valproic acid. J Biomed Biotechnol. 2010;2010:479364. doi:10.1155/2010/479364

11. Verrotti A, Scaparrotta A, Grosso S, Chiarelli F, Coppola G. Anticonvulsant drugs and hematological disease. Neurol Sci. 2014;35(7):983-993. doi:10.1007/s10072-0141701-0

12. Kurwale N, Garg K, Arora A, Chandra PS, Tripathi M. Valproic acid as an antiepileptic drug: Is there a clinical relevance for the epilepsy surgeon?. Epilepsy Res. 2016;127:191-194. doi:10.1016/j.eplepsyres.2016.09.005

13. Abdallah $\mathrm{C}$. Considerations in perioperative assessment of valproic acid coagulopathy. $\mathrm{J}$ Anaesthesiol Clin Pharmacol. 2014;30(1):7-9. doi:10.4103/0970-9185.125685

14. Allarakhia IN, Garofalo EA, Komarynski MA, Robertson PL. Valproic acid and thrombocytopenia in children: a case-controlled retrospective study. Pediatr Neurol. 1996;14(4):303-307. doi:10.1016/08878994(96)00052-5

15. Gidal B, Spencer N, Maly M, et al. Valproatemediated disturbances of hemostasis: relationship to dose and plasma concentration. Neurology. 1994;44(8):1418-1422. doi:10.1212/wnl.44.8.1418 